\title{
Rapid Centrifugation in the Routine Hemostasis Laboratory
}

\author{
Nathan Wolfensberger ${ }^{1, *}$ Georgios Georgiou ${ }^{2, *}$ Evelyne Giabbani $^{3}$ Marianne Reusser ${ }^{3}$ \\ Linet M. Njue ${ }^{3}$ Martin Fiedler ${ }^{4}$ Alexander B. Leichtle ${ }^{4,5}$ Michael Nagler ${ }^{3,4}$
}

\footnotetext{
${ }^{1}$ Department of Medical Oncology and Hematology, University Hospital Zurich, Zurich, Switzerland

2 Unilabs Lausanne, Lausanne, Switzerland

${ }^{3}$ Department of Hematology and Central Hematology Laboratory, Inselspital, Bern University Hospital, University of Bern, Bern, Switzerland

${ }^{4}$ University Institute of Clinical Chemistry, Inselspital, Bern University Hospital, University of Bern, Bern, Switzerland

${ }^{5}$ Insel Data Science Center, Inselspital, Bern University Hospital, University of Bern, Bern, Switzerland
}

Thromb Haemost 2019;119:2025-2033.

\begin{abstract}
Address for correspondence Michael Nagler, MD, PhD, MSc, University Institute of Clinical Chemistry, Inselspital, Bern University Hospital, 3010 Bern, Switzerland (e-mail: michael.nagler@insel.ch).
\end{abstract}

\section{Abstract}

\section{Keywords}

- centrifugation

- automation

- laboratory/ instrumentation

- laboratory/methods

- laboratory/standards

- blood coagulation tests
Background The use of short and uniform centrifugation schemes contributes significantly to the successful automation of laboratory procedures. It is however unclear if this is applicable to the hemostasis laboratory.

Objectives This article assesses the accuracy of measurements obtained with a rapid, high-speed centrifugation scheme in a large set of hemostasis tests, covering the full spectrum of values obtained in clinical practice, and using meaningful statistical measures.

Methods Two citrated plasma samples were obtained from consecutive patients of a tertiary hospital with suspected abnormal hemostasis tests and processed with two centrifugation schemes in parallel: $1,500 \times g$ for 10 minutes and $3,137 \times g$ for 7 minutes. The following tests were conducted: prothrombin time $(n=125)$, international normalized ratio $(n=146)$, activated partial thromboplastin time $(n=119)$, thrombin time $(n=105)$, fibrinogen $(n=125)$, factor $(\mathrm{F}) \|(n=69)$, FV $(n=64)$, FVII $(n=64), \mathrm{FX}(n=67)$, FVIII $(n=55)$, FIX $(n=37), \mathrm{FXI}(n=35)$, and FXIII $(n=20)$, D-dimer $(n=34)$, antithrombin $(n=31)$, anti-Xa activity $(n=30)$, von Willebrand antigen $(n=25)$, and von Willebrand activity (VWF:GPIbM; $n=27)$.

Results A wide range of results were obtained in all tests. Spearman's rank correlation coefficient was at least 0.95 for all tests except FV, FIX, and FXI. The coverage probability $\pi$ at a given deviation index $\mathrm{k}$ of $15 \%$ was above 0.9 for all tests except FV, FVII, FX, FVIII, FIX, FXI, and VWF:GPIbM, suggesting a lack of agreement.

Conclusion Our results suggest that high-speed centrifugation is applicable to the majority of routine hemostasis parameters. The coverage probability was more sensitive than Spearman's rank correlation to detect disagreement among centrifugation schemes.

* These authors contributed equally to this study.

received

June 14, 2019

accepted after revision July 21, 2019
DOI https://doi.org/

10.1055/s-0039-1696712. ISSN 0340-6245. (c) 2019 Georg Thieme Verlag KG Stuttgart · New York
License terms

()(1) $\odot \circledast$ 


\section{Introduction}

Total laboratory automation (TLA) is seen as an important strategy to tackle today's challenges in laboratory medicine and related health care. ${ }^{1-6}$ Integrating diverse analyzers of multiple specialities as well as preanalytic and sorting modules to one track can replace many manual tasks and save labor., ${ }^{4,7}$ TLA can save a relevant amount of long-term health care costs despite high initial investments. ${ }^{8}$ The efficiency can be improved by reducing the turnaround time (TAT) and increasing the throughput. ${ }^{9,10}$ Risky and laborious manual sample management can be avoided by algorithms identifying samples and triggering rerun or reflex tests, thus improving accuracy of results. ${ }^{4}$ Controlling all phases of the analytical process using software programs run centrally enhances standardization, supports accreditation procedures, and improves quality in general. ${ }^{4}$ One important requirement for the integration of analyzers and specialities into TLA is uniform and ideally rapid centrifugation schemes. This might prevent delay of samples on the track and improve total TAT.

It is however uncertain whether rapid centrifugation schemes-used in clinical chemistry as well as immunochemistry-can be applied in the hemostasis laboratory. Coagulation-based assays are considered to be influenced by many preanalytic factors. ${ }^{11}$ The residual platelet count after centrifugation is regarded as the most important influencing factor ${ }^{12}$ and the platelet count is subject to the centrifugal forces applied. ${ }^{13}$ Mechanical stimulation is discussed as a contributing factor as well. ${ }^{14}$ Some authors recommend avoiding centrifugal forces above $1,500 \times \mathrm{g}^{15}$ but others do not. $^{16,17}$ Several scientific societies in the United States (Clinical and Laboratory Standards Institute [CLSI ${ }^{18}$ ), France (Groupe d'Etude sur l'Hémostase et la Thrombose ${ }^{19}$ ), and the United Kingdom (British Committee for Standards in Haematology ${ }^{20}$ ) as well as other authors ${ }^{11}$ have released guidance documents and recommend centrifugation schemes that aim to achieve a platelet count below $10 \times 10^{\wedge} 9 /$ L using $1,500 \times g$ for 10 to 15 minutes. Whether this can be accelerated using higher centrifugal forces remains unclear. Limitations of previous investigations include (1) a limited number of patients or samples, (2) a paucity of hemostasis tests studied, (3) a very limited spectrum of test results covered (normal only), (4) nonstandard centrifugation schemes as comparators, and (5) use of insensitive statistical measures.

With the present study, we aimed to comprehensively assess the accuracy of a rapid, high-speed centrifugation scheme compared with an established and recommended centrifugation scheme in a comprehensive set of hemostasis tests, covering a large spectrum of values, and using meaningful statistical measures.

\section{Methods}

\section{Study Design, Setting, and Population}

This prospective cohort study was conducted in a university hospital (Inselspital, Bern University Hospital, Bern, Switzerland) between October 2015 and March 2016. We included consecutive patients from a variety of departments (cardiovascular surgery, internal medicine, intensive care unit, and hemophilia outpatient unit). The inclusion criterion was "suspected abnormal hemostasis laboratory test result" to obtain a sample set covering a broad spectrum of results. Exclusion criteria were an age below 18 years and refused general consent. Patients were screened by treating physicians in the respective departments. The protocol was approved by the local ethics committee (Kantonale Ethikkommission Bern; March 18, 2014) and all patients signed the general consent of the hospital.

\section{Collection and Handling of Samples}

An in-house protocol for blood drawing was implemented at our institution to ensure adequate preanalytic conditions. ${ }^{18}$ Plasma samples were collected after ethylenediaminetetraacetic acid (EDTA) and serum samples to avoid contamination with residual heparin or tissue factor if blood was accidentally obtained from a vascular access device or using a butterfly needle. $^{12,19,21}$ Venous blood was drawn into two plastic syringes of $4.5 \mathrm{~mL}$ containing $0.106 \mathrm{~mol} / \mathrm{L}$ trisodium citrate (Monovette, Sarstedt, Nümbrecht, Germany). Samples were transported immediately to the central laboratory and the handling of both samples was identical.

\section{Centrifugation Schemes}

Both plasma samples were centrifuged within 15 minutes after arrival in the laboratory. The established standard centrifugation scheme in our institution was $1,500 \times \mathrm{g}$ for 10 minutes (established after determining residual platelet count in 30 samples). ${ }^{11,19,20}$ The high-speed centrifugation scheme was defined as $3,137 \times g$ for 7 minutes $(3,137 \times g$ is the established speed in clinical chemistry at our institution and the time of 7 minutes was chosen after conducting a pilot study analyzing residual platelet concentration). Z500 centrifuges were used (HERMLE Labortechnik GmbH, Wehingen, Germany), implemented in a GLP laboratory track (GLP Systems GmbH, Hamburg, Germany). The centrifuges work at $20^{\circ} \mathrm{C}$ using a brake. After centrifugation, samples were transferred into aliquots and snap-frozen at $-20^{\circ} \mathrm{C}$. Samples were transferred to $-80^{\circ} \mathrm{C}$ if not analyzed within 1 to 2 weeks. All hemostasis parameters were determined within 2 months following centrifugation.

\section{Determination of Laboratory Assays}

Platelet count was analyzed using an automated hematology analyzer (Coulter Counter LH750, Beckman-Coulter Inc., Nyon, Switzerland). Prothrombin time was measured using Dade Innovin reagent (Siemens Healthcare Diagnostics Products GmbH, Marburg, Germany) on a Sysmex CS 5100 analyzer (distributed by Siemens Healthcare Diagnostics Products $\mathrm{GmbH}$ ) and expressed as prothrombin ratio (PR, \%). Activated partial thromboplastin time (aPTT) was determined using Pathrombin SL (Siemens Healthcare Diagnostics Products $\mathrm{GmbH}$ ) on a Sysmex CS 5100. Thrombin time was determined using Thromboclotin reagent (Siemens Healthcare Diagnostics Products $\mathrm{GmbH}$ ) on a Sysmex CS 5100. D-dimers were measured immunoturbidimetrically by Innovance D-Dimer 
(Siemens Healthcare Diagnostics Products $\mathrm{GmbH}$ ) on a Sysmex CS 5100. Functional antithrombin activity was determined using INNOVANCE Antithrombin (Siemens Healthcare Diagnostics Products $\mathrm{GmbH}$ ) on a Sysmex CS 5100. Fibrinogen was assessed in plasma by the Clauss method using Dade Thrombin Reagent (Siemens Healthcare Diagnostics Products $\mathrm{GmbH}$ ) on a Sysmex CS 5100. Factor (F)II, FV, FVII, and FX were measured by a one-stage coagulometric assay using Dade Innovin and factordeficient plasma (Siemens Healthcare Diagnostics Products $\mathrm{GmbH}$ ) on a Sysmex CS 5100. FVIII, FIX, and FXI were determined using a one-stage coagulometric assay using Pathrombin SL and factor-deficient plasma on a Siemens BCS XP analyzer (Siemens Healthcare Diagnostics Products GmbH). FXIII was analyzed chromatographically using Berichrom Factor XIII test kit (Siemens Healthcare Diagnostics Products $\mathrm{GmbH}$ ) on a Siemens BCS XP. von Willebrand factor antigen (VWF:Ag) was determined using the VWF Ag Testkit (Siemens Healthcare Diagnostics Products GmbH) and activity (VWF:GPIbM) using Siemens Innovance VWF Ac (Siemens Healthcare Diagnostics Products $\mathrm{GmbH}$ ). Chromogenic anti-Xa activity for monitoring unfractionated heparin (UFH) as well as low molecular weight heparin (LMWH) was determined using BIOPHEN Heparin LRT (HYPHEN BioMed, Neuville-sur-Oise, France) on a Sysmex CS 5100.

To contextualize these measurements, we determined the within-run and day-to-day imprecision in two samples (one with results in the normal range [n] and one with results in the abnormal range [p]), 10 times each.

\section{Statistical Analysis}

Median (interquartile range, IQR) and numbers (percentages) were used for descriptive purposes. To analyze concordance between the two centrifugation schemes, we determined the Spearman's rank correlation coefficient as well as the coverage probability (CP $\pi$ ). The CP $\pi$ outlines agreement with several advantages compared with more traditional measures: the computation is simple, discordant measurements can be identified rapidly, the interpretation is intuitive, and it can be applied to continuous as well as categorical data in a broad spectrum of observers and populations. ${ }^{22}$ The $\mathrm{CP} \pi$ can be interpreted as the proportion of observations falling within the paired absolute difference $\mathrm{\kappa}$, a priori defined as acceptable (the tolerable total deviation index, TDI). In our study, we defined a TDI of 0.15 as acceptable. The tolerable CP $\pi$ was assumed at 0.9. Bland-Altman difference plots for determination of bias as well as lower and higher limits of agreement were additionally created. Coefficients of variation were calculated for within-run and day-to-day imprecision. Analyses were restricted to observations in a meaningful range: PR, 0 to $110 \%$; international normalized ratio (INR), 0.9 to 5.0 ; thrombin time, 10 to 70 seconds; aPTT, 15 to 100 seconds; fibrinogen, 0 to $6.0 \mathrm{~g} / \mathrm{L}$; D-dimer, 0 to 2,000 $\mu \mathrm{g} / \mathrm{L}$; antithrombin, 0 to $120 \%$; FII, FV, FVII, FX: 0 to $120 \%$; FVIII, FIX, FXI, FXIII: 0 to $200 \%$; and VWF: GPIbM and VWF:Ag: 0 to 200\%. In addition, LMWH measurements below $0.1 \mathrm{U} / \mathrm{mL}$ and D-dimer below $400 \mathrm{U} / \mathrm{mL}$ were censored for CP measurements. The Stata 14.1 statistics software package (StataCorp. 2015; Stata Statistical Software: Release 14.2, StataCorp LP, College Station, Texas, United States) as well as R ( R Development Core Team [2008]; R: A language and environment for statistical computing; $R$ Foundation for Statistical Computing, Vienna, Austria; ISBN 3-900051-07-0, http://www.R-project.org) were used for statistical analyses. Figures were created using Prism 6 (GraphPad Software, Inc., La Jolla, California, United States).

\section{Results}

\section{Patients}

Samples from 198 patients were analyzed, the flow is illustrated in - Fig. 1. Seventy-four patients were recruited from hematology outpatient unit (37\%), 51 patients from intensive care unit (26\%), 48 patients from general internal medicine (24\%), and 25 patients from the surgery department (13\%). The median age of the patients was 56.0 years (range: $19.7-94.4)$ and $47.5 \%$ were female $(n=94)$. The number of observations per test is shown in -Table $\mathbf{1}$. The range of values is reported in - Table $\mathbf{1}$, the distribution is illustrated in scatterplots (-Supplementary Figs. S1-S3, available in the online version).

\section{Platelet count}

The median platelet count in plasma after centrifugation with $3,137 \times g$ for 7 minutes was $8 \times 10^{\wedge} 9 / \mathrm{L}$ (IQR: $4-12 \times 10^{\wedge} 9 / \mathrm{L}$; $n=30$ ). Platelet count was $6 \times 10^{\wedge} 9 / \mathrm{L}$ (IQR: $3-9 ; n=30$ ) in samples centrifuged at $1,500 \times g$ for 10 minutes.

\section{Concordance between Centrifugation Schemes}

All results are reported in detail in - Table 1. Scattergrams of PR, aPTT, fibrinogen, and D-dimer are shown as an example in - Fig. 2. Scattergrams and Bland-Altman difference plots for all tests are shown in -Supplementary Figs. S1-S3 (available in the online version). - Fig. 3 summarizes the accuracy of all tests and contrasts $r_{\mathrm{s}}$ to CP estimates.

With regard to the routine parameters $P T$, INR, thrombin time, and $a P T T$, correlation $\left(r_{\mathrm{s}}\right)$ was clearly above 0.95 and $\mathrm{CP}$ $\pi$ above 0.9 (-Table 1). Bias and limits of agreement of the Bland-Altman difference plot were close to zero. Accordingly, coefficient of variation (CV) was below $5 \%$ with regard to within-run and day-to-day imprecision (day-to-day imprecision of the abnormal sample: $6.7 \%$ ).

Similar results were observed with regard to fibrinogen, $D$-dimer, and antithrombin activity measurements: $r_{\mathrm{s}}$ was above 0.95 , CP $\pi$ above 0.9 , and Bland-Altman estimates close to zero. CV was below 5\% (except within-run imprecision of the normal D-dimer sample: $12.0 \%$ ).

Anti-Xa activity measurements (UFH and LMWH) resulted in $r_{\mathrm{s}}$ above 0.95 and a CP $\pi$ above 0.9 as well. Bland-Altman estimates were close to zero and CV was below 5\% (except day-to-day imprecision of LMWH-measurements: $5.9 \%$ ).

Correlation $\left(r_{\mathrm{s}}\right)$ was above 0.95 and $\mathrm{CP} \pi$ above 0.9 for $V W F$ : Ag but not for VWF:GPIbM (0.76; 95\% confidence interval: $0.59-0.89$ ). Accordingly, the lower limit of agreement was -18.1 , and the higher limit of agreement was 10 for VWF:GPIbM. Within-run as well as day-to-day imprecision was below $5 \%$.

A borderline $r_{\mathrm{s}}$ was observed for FV (92\%), FVII (95\%), and FX (95\%), the CP $\pi$ was clearly below 0.9 (see - Table 1). 
2028 Rapid Centrifugation in the Routine Hemostasis Laboratory Wolfensberger et al.

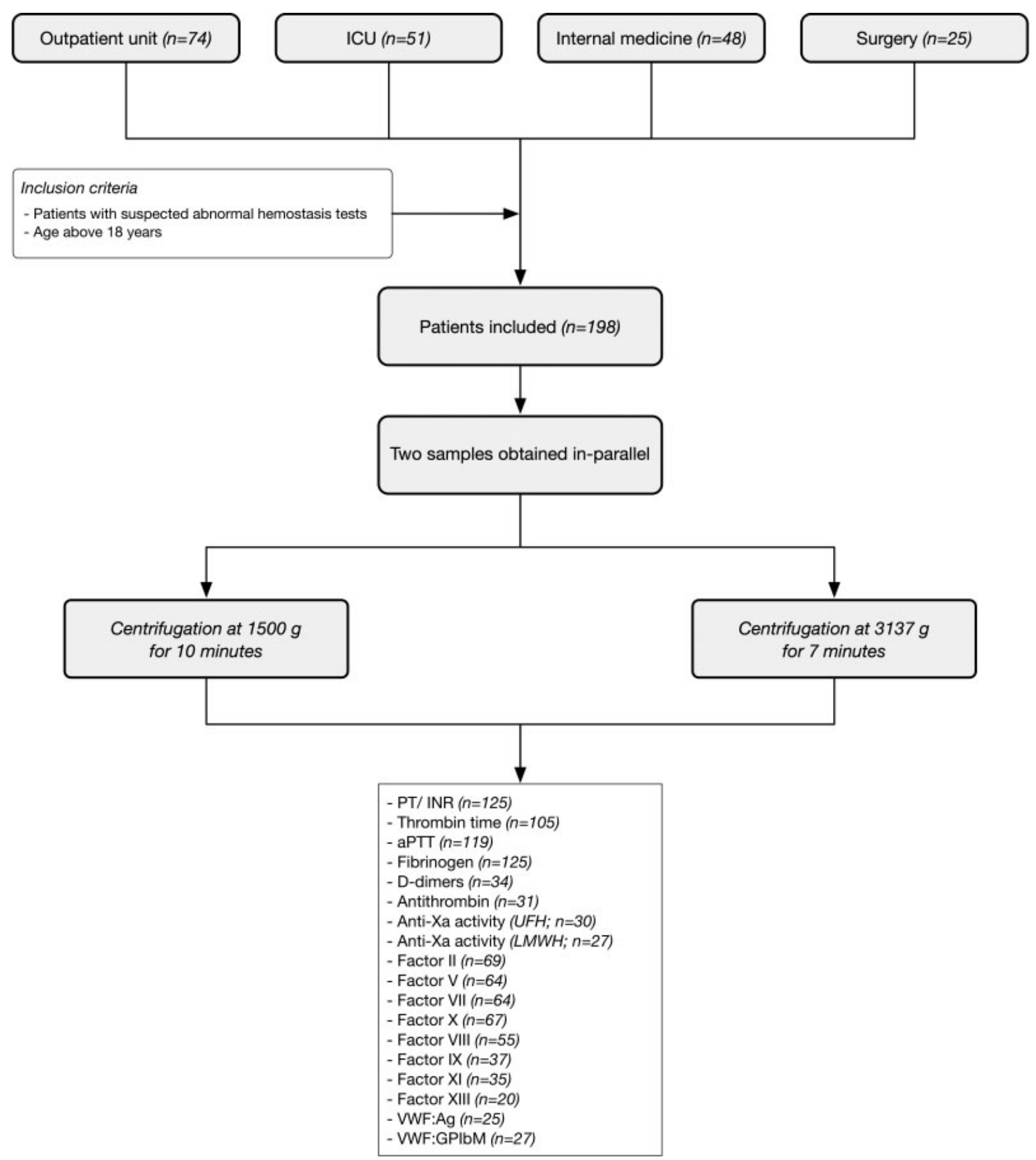

Fig. 1 Sample flow.

Accordingly, the agreement interval of the Bland-Altman plot was wide. With regard to FII, $r_{\mathrm{s}}$ was 0.97 and $\mathrm{CP} \pi 0.95$. Within-run as well as day-to-day imprecision was low $(\mathrm{CV}<5 \%$ for $F V I I$, and $<10 \%$ for $F I I, F V$, and $F X)$.

In the case of FVIII, $r_{\mathrm{s}}$ was 0.96 but $\mathrm{CP} \pi$ was $0.30 ; r_{\mathrm{s}}$ was below 0.95 and $\mathrm{CP} \pi$ below 0.9 in the case of FIX as well as FXI. The agreement interval of the Bland-Altman plot was wide (-Table 1). Within-run as well as day-to-day imprecision was below $10 \%$.
With regard to FXIII, $r_{\mathrm{s}}$ was 0.96 and $\mathrm{CP} \pi 0.96$; CV was below $10 \%$.

\section{Discussion}

In the present study, we tested the accuracy of measurements obtained with rapid, high-speed centrifugation scheme studying a large spectrum of hemostasis tests and covering the full range of values obtained in clinical practice. 
Table 1 Concordance results of routine hemostasis measurements obtained with a rapid, high-speed centrifugation scheme in comparison to a traditional scheme

\begin{tabular}{|c|c|c|c|c|c|c|c|c|c|}
\hline \multirow[t]{2}{*}{ Parameter } & \multirow[t]{2}{*}{ Obs. } & \multirow[t]{2}{*}{ Results } & \multirow{2}{*}{$\begin{array}{l}\begin{array}{l}\text { Correlation } \\
\text { coefficient }\end{array} \\
r_{\mathrm{s}}\end{array}$} & \multirow{2}{*}{$\begin{array}{l}\text { Coverage } \\
\text { probability } \pi^{a}\end{array}$} & \multicolumn{4}{|c|}{ Bland-Altman difference plot } & \multirow{2}{*}{$\begin{array}{l}\begin{array}{l}\text { Analytical } \\
\text { variability }\end{array} \\
\begin{array}{l}\text { Day-to-day } \\
\text { imprecision }\end{array}\end{array}$} \\
\hline & & & & & Bias & LLA & HLA & $\begin{array}{l}\text { Within-run } \\
\text { imprecision }\end{array}$ & \\
\hline & Numbers & $\begin{array}{l}\text { Median } \\
\text { (range) }\end{array}$ & $(95 \% \mathrm{Cl})$ & $(95 \% \mathrm{Cl})$ & $(95 \% \mathrm{Cl})$ & & & (CV in \%) & (CV in \%) \\
\hline PR (\%) & 125 & $\begin{array}{l}78.5 \\
(6.6,107.8)\end{array}$ & $\begin{array}{l}0.99 \\
(0.98,0.99)\end{array}$ & $\begin{array}{l}0.99 \\
(0.84,1.00)\end{array}$ & $\begin{array}{l}0.2 \\
(-0.3,0.7)\end{array}$ & -5.6 & 6.0 & $\begin{array}{l}0.6(n) \\
0.8(p)\end{array}$ & $\begin{array}{l}4.0(n) \\
2.2(p)\end{array}$ \\
\hline INR & 146 & $\begin{array}{l}1.04 \\
(0.9,3.5)\end{array}$ & $\begin{array}{l}0.99 \\
(0.99,0.99)\end{array}$ & $\begin{array}{l}1.00 \\
(0.96,1.00)\end{array}$ & $\begin{array}{l}0.0 \\
(-0.0,0.0)\end{array}$ & -0.2 & 0.2 & $\begin{array}{l}0.7(n) \\
0.9(p)\end{array}$ & $\begin{array}{l}4.2(n) \\
2.4(p)\end{array}$ \\
\hline $\begin{array}{l}\text { Thrombin } \\
\text { time (s) }\end{array}$ & 105 & $\begin{array}{l}16.5 \\
(13.2,57.3)\end{array}$ & $\begin{array}{l}0.97 \\
(0.95,0.98)\end{array}$ & $\begin{array}{l}1.00 \\
(1.00,1.00)\end{array}$ & $\begin{array}{l}-0.1 \\
(-0.3,0.0)\end{array}$ & -1.6 & 1.4 & $\begin{array}{l}2.0(n) \\
2.9(p)\end{array}$ & $\begin{array}{l}2.2(n) \\
2.6(p)\end{array}$ \\
\hline aPTT (s) & 119 & $\begin{array}{l}34.9 \\
(22.0,96.0)\end{array}$ & $\begin{array}{l}0.99 \\
(0.99,0.99)\end{array}$ & $\begin{array}{l}1.00 \\
(1.00,1.00)\end{array}$ & $\begin{array}{l}0.0 \\
(-0.2,0.2)\end{array}$ & -2.1 & 2.1 & $\begin{array}{l}0.6(n) \\
1.1(p)\end{array}$ & $\begin{array}{l}1.8(n) \\
6.7(p)\end{array}$ \\
\hline $\begin{array}{l}\text { Fibrinogen } \\
(\mathrm{g} / \mathrm{L})\end{array}$ & 125 & $\begin{array}{l}2.3 \\
(0.0,5.8)\end{array}$ & $\begin{array}{l}0.98 \\
(0.97,0.99)\end{array}$ & $\begin{array}{l}0.96 \\
(0.91,0.99)\end{array}$ & $\begin{array}{l}-0.0 \\
(-0.0,0.0)\end{array}$ & -0.3 & 0.3 & $\begin{array}{l}1.2(\mathrm{n}) \\
1.1(\mathrm{p})\end{array}$ & $\begin{array}{l}3.2(n) \\
6.0(p)\end{array}$ \\
\hline $\begin{array}{l}\text { D-dimer } \\
(\mu \mathrm{g} / \mathrm{L})\end{array}$ & 34 & $\begin{array}{l}395 \\
(17,1970) \\
\end{array}$ & $\begin{array}{l}0.99 \\
(0.99,1.00) \\
\end{array}$ & $\begin{array}{l}1.00 \\
(0.99,1.00) \\
\end{array}$ & $\begin{array}{l}13.4 \\
(3.5,23.4) \\
\end{array}$ & -43.4 & 70.3 & $\begin{array}{l}12.0(n) \\
2.0(p)\end{array}$ & $\begin{array}{l}3.0(n) \\
2.0(p)\end{array}$ \\
\hline $\begin{array}{l}\text { Antithrombin } \\
\text { (\%) }\end{array}$ & 31 & $\begin{array}{l}76.6 \\
(40.4,108.5) \\
\end{array}$ & $\begin{array}{l}0.99 \\
(0.99,1.00) \\
\end{array}$ & $\begin{array}{l}1.00 \\
(0.99,1.00) \\
\end{array}$ & $\begin{array}{l}0.2 \\
(-0.7,1.1) \\
\end{array}$ & -4.7 & 5.1 & $\begin{array}{l}1.3(\mathrm{n}) \\
2.8(\mathrm{p})\end{array}$ & $\begin{array}{l}1.1(\mathrm{n}) \\
2.2(\mathrm{p}) \\
\end{array}$ \\
\hline $\begin{array}{l}\text { Anti-Xa activity } \\
\text { (UFH; } \mathrm{U} / \mathrm{mL} \text { ) }\end{array}$ & 30 & $\begin{array}{l}0.10 \\
(0.01,0.92) \\
\end{array}$ & $\begin{array}{l}0.99 \\
(0.98,1.00) \\
\end{array}$ & $\begin{array}{l}0.94 \\
(0.86,0.99) \\
\end{array}$ & $\begin{array}{l}0.00 \\
(-0.01,0.01) \\
\end{array}$ & -0.03 & 0.03 & $2.1(p)$ & $3.8(p)$ \\
\hline $\begin{array}{l}\text { Anti-Xa activity } \\
\text { (LMWH; U/mL) }\end{array}$ & 27 & $\begin{array}{l}0.06 \\
(0.00,0.51)\end{array}$ & $\begin{array}{l}0.99 \\
(0.97,0.99)\end{array}$ & $\begin{array}{l}0.93 \\
(0.77,1.00)\end{array}$ & $\begin{array}{l}0.00 \\
(-0.01,0.01)\end{array}$ & -0.03 & 0.03 & $1.7(p)$ & $5.9(p)$ \\
\hline Factor II (\%) & 69 & $\begin{array}{l}84.0 \\
(16.0,119.0)\end{array}$ & $\begin{array}{l}0.97 \\
(0.96,0.98)\end{array}$ & $\begin{array}{l}0.95 \\
(0.81,1.00)\end{array}$ & $\begin{array}{l}0.6 \\
(-0.6,1.8)\end{array}$ & -9.2 & 10.4 & $\begin{array}{l}1.4(n) \\
1.9(p)\end{array}$ & $\begin{array}{l}1.8(n) \\
6.9(p)\end{array}$ \\
\hline Factor V (\%) & 64 & $\begin{array}{l}85.0 \\
(0.0,120.0)\end{array}$ & $\begin{array}{l}0.92 \\
(0.86,0.95)\end{array}$ & $\begin{array}{l}0.41 \\
(0.20,0.65)\end{array}$ & $\begin{array}{l}-1.6 \\
(-4.3,1.1)\end{array}$ & -23.3 & 20.2 & $\begin{array}{l}2.2(\mathrm{n}) \\
2.4(\mathrm{p})\end{array}$ & $\begin{array}{l}4.1(n) \\
5.2(p)\end{array}$ \\
\hline Factor VII (\%) & 64 & $\begin{array}{l}65.3 \\
(15.0,120.0)\end{array}$ & $\begin{array}{l}0.95 \\
(0.92,0.97)\end{array}$ & $\begin{array}{l}0.57 \\
(0.36,0.84)\end{array}$ & $\begin{array}{l}-1.1 \\
(-3.2,0.9)\end{array}$ & -17.5 & 15.3 & $\begin{array}{l}1.6(n) \\
3.4(p)\end{array}$ & $\begin{array}{l}2.9(n) \\
3.3(p)\end{array}$ \\
\hline Factor X (\%) & 67 & $\begin{array}{l}75.1 \\
(5.0,118.8)\end{array}$ & $\begin{array}{l}0.95 \\
(0.91,0.97)\end{array}$ & $\begin{array}{l}0.60 \\
(0.46,0.74)\end{array}$ & $\begin{array}{l}-0.4 \\
(-2.4,1.7)\end{array}$ & -17.2 & 16.5 & $\begin{array}{l}1.9(n) \\
1.4(p)\end{array}$ & $\begin{array}{l}3.0(n) \\
5.6(p)\end{array}$ \\
\hline Factor VIII (\%) & 55 & $\begin{array}{l}79.5 \\
(0.3,198.0)\end{array}$ & $\begin{array}{l}0.96 \\
(0.93,0.98)\end{array}$ & $\begin{array}{l}0.30 \\
(0.21,0.38)\end{array}$ & $\begin{array}{l}-17.5 \\
(-22.3,-12.7)\end{array}$ & -53.0 & 17.9 & $\begin{array}{l}2.8(n) \\
2.3(p)\end{array}$ & $\begin{array}{l}5.5(n) \\
4.0(p)\end{array}$ \\
\hline Factor IX (\%) & 37 & $\begin{array}{l}104.0 \\
(15.0,138.0)\end{array}$ & $\begin{array}{l}0.84 \\
(0.70,0.91)\end{array}$ & $\begin{array}{l}0.79 \\
(0.61,0.90)\end{array}$ & $\begin{array}{l}-0.9 \\
(-4.5,2.7)\end{array}$ & -22.5 & 20.7 & $\begin{array}{l}1.9(n) \\
2.1(p)\end{array}$ & $\begin{array}{l}3.3(n) \\
6.2(p)\end{array}$ \\
\hline Factor XI (\%) & 35 & $\begin{array}{l}96.0 \\
(47.0,163.0)\end{array}$ & $\begin{array}{l}0.79 \\
(0.61,0.89)\end{array}$ & $\begin{array}{l}0.80 \\
(0.68,0.90)\end{array}$ & $\begin{array}{l}-1.3 \\
(-4.8,2.2)\end{array}$ & -21.6 & 19.0 & $\begin{array}{l}3.1(\mathrm{n}) \\
1.8(\mathrm{p})\end{array}$ & $\begin{array}{l}2.6(n) \\
7.3(p)\end{array}$ \\
\hline Factor XIII (\%) & 20 & $\begin{array}{l}110.5 \\
(15.1,162.0)\end{array}$ & $\begin{array}{l}0.96 \\
(0.89,0.98)\end{array}$ & $\begin{array}{l}0.96 \\
(0.82,0.99)\end{array}$ & $\begin{array}{l}-2.5 \\
(-5.9,1.0)\end{array}$ & -17.0 & 12.1 & $\begin{array}{l}3.2(n) \\
2.0(p)\end{array}$ & $\begin{array}{l}5.6(n) \\
3.8(p)\end{array}$ \\
\hline VWF:Ag (\%) & 25 & $\begin{array}{l}87.0 \\
(12.0,191.3)\end{array}$ & $\begin{array}{l}0.99 \\
(0.97,0.99)\end{array}$ & $\begin{array}{l}0.96 \\
(0.83,0.99)\end{array}$ & $\begin{array}{l}-1.2 \\
(-2.9,0.5)\end{array}$ & -9.4 & 7.0 & $\begin{array}{l}1.7(n) \\
2.4(p)\end{array}$ & $\begin{array}{l}1.9(n) \\
4.1(p)\end{array}$ \\
\hline $\begin{array}{l}\text { VWF:GPIbM } \\
(\%)\end{array}$ & 27 & $\begin{array}{l}84.0 \\
(7.0,178.9)\end{array}$ & $\begin{array}{l}0.98 \\
(0.95,0.99)\end{array}$ & $\begin{array}{l}0.76 \\
(0.59,0.89)\end{array}$ & $\begin{array}{l}-3.8 \\
(-6.6,-1.0)\end{array}$ & -18.1 & 10.4 & $\begin{array}{l}1.7(\mathrm{n}) \\
1.6(p)\end{array}$ & $\begin{array}{l}2.2(n) \\
2.7(p)\end{array}$ \\
\hline
\end{tabular}

Abbreviations: aPTT, activated partial thromboplastin time; CV, coefficient of variation; HLA, higher limit of agreement; INR, international normalized ratio; LLA, lower limit of agreement; LMWH, low molecular weight heparin; Obs., observations; PR, prothrombin ratio; $r_{s}$, Spearman's rank correlation coefficient; UFH, unfractionated heparin; VWF:Ag, von Willebrand factor antigen; VWF:GPIbM, von Willebrand factor functional activity as measured using the spontaneous binding of VWF to a gain-of-function mutant GPIb fragment.

${ }^{a}$ Based on an accepted total deviation index of 0.15 .

bobtained in two samples, one with results in the normal range [n] and one with results in the abnormal range [p], 10 times each.

$\mathrm{CP} \pi$ identified more assays with nonconcordant results than Spearman's correlation coefficient. Whereas an appropriate concordance between measurements were observed for all routine parameters, relevant discrepancies were observed with regard to most single-factor determinations as well as VWF:GPIbM.
Our observations and data reported here are in line with the limited data published to date on this topic. Lippi et al compared samples obtained in 15 healthy volunteers and centrifuged with $3,000 \times g$ versus $1,500 \times g$. Significant differences were found with regard to FVIII and IX, but not for PT, aPTT, or fibrinogen. ${ }^{13}$ Several routine tests (fibrinogen, 
A

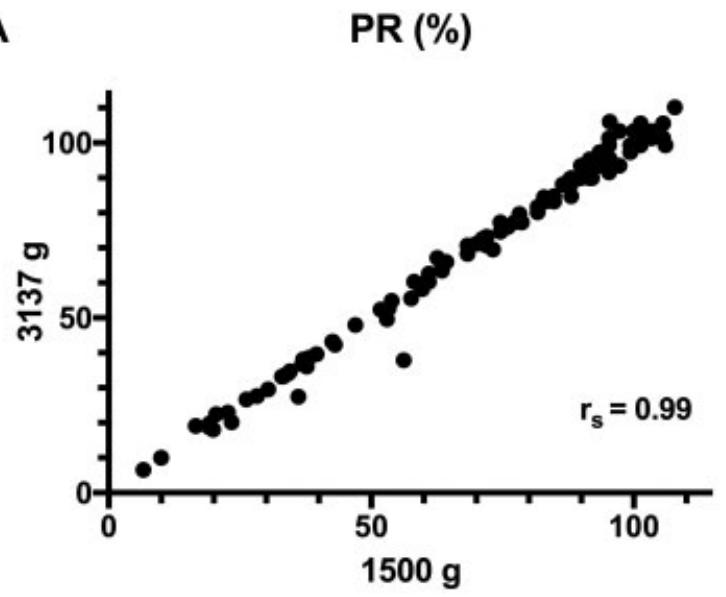

C

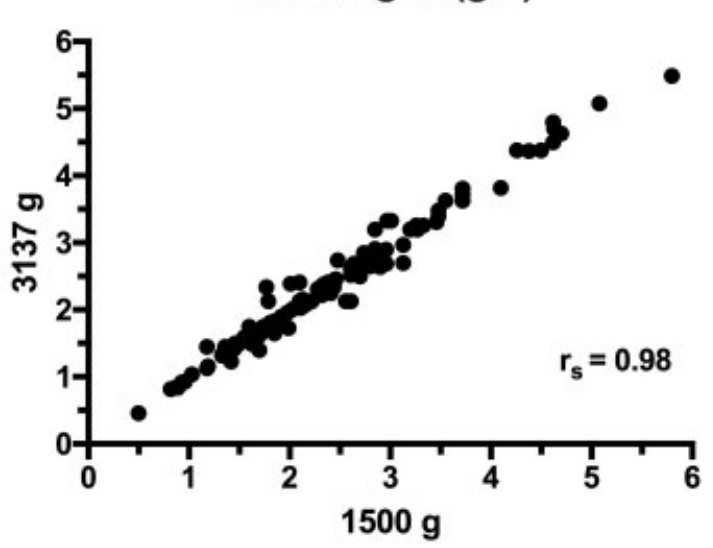

B

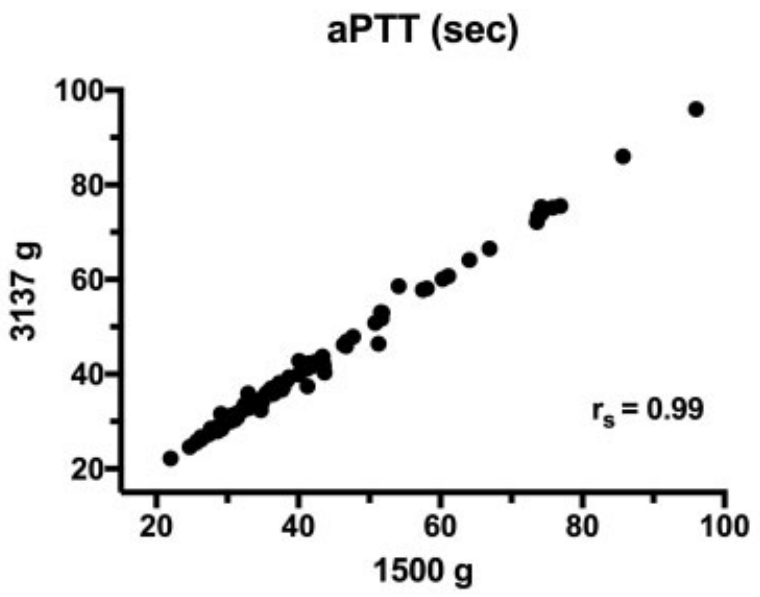

D

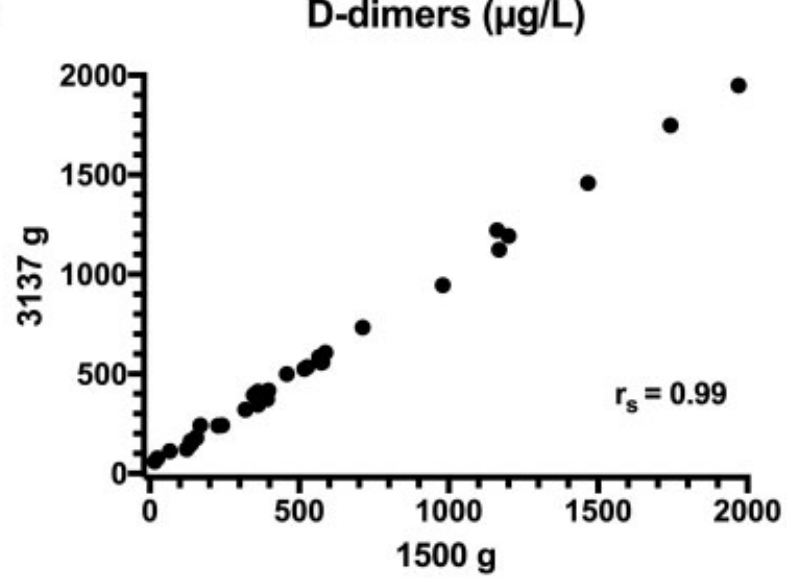

Fig. 2 Concordance of routine hemostasis measurements obtained with a rapid, high-speed centrifugation scheme in comparison to a traditional scheme. Scattergrams of prothombin ratio (PR, \%), activated partial thromboplastin time (aPTT, seconds), fibrinogen (Clauss method; $\mathrm{g} / \mathrm{L}$ ), and D-dimer $(\mu \mathrm{g} / \mathrm{L})$ are shown as an example, all other results are shown in the - Supplementary Material. Spearman's rank correlation coefficient $\left(r_{s}\right)$ is reported.

INR, aPTT, D-dimer) as well as single factors have also been studied $(1,500 \times g$ for 5 minutes vs. $3,000 \times g$ for 7 minutes $)$ in a limited number of patients with mostly normal test results and a correlation coefficient of all routine tests as well as most factors studies was above $0.9 .^{23}$ Suchsland et al have compared PT, aPTT, FVIII, and protein S in one versus two runs of $3,280 \times g$ for 5 minutes. The outcome of that work reported a correlation coefficient above 0.9 for all but protein $\mathrm{S}^{24}$ Boissier et $\mathrm{al}^{17}$ compared a centrifugal spin protocol of $4,500 \times g$ for 2 minutes to a $2,200 \times g$ for 15 minutes protocol in 200 patients in the following assays PT, INR, aPTT, fibrinogen, thrombin time, FII, FV, antithrombin, and anti-Xa activity. ${ }^{17}$ These authors concluded that the agreement was adequate. We have also essentially repeated the Boissier centrifugation protocols in samples of patients treated with direct oral anticoagulations and measurements of anti-Xa activity, and we have arrived at similar conclusions. ${ }^{16}$ In another study, PT, aPTT, and INR measurements were compared in platelet-poor plasma and platelet-rich plasma of 100 samples, and again found to be similar. ${ }^{21}$

\section{Strengths and Limitations/Implications}

In contrast to previous studies, we have studied a broad range of hemostasis tests covering the full spectrum of test results in a large number of patients recruited in clinical practice. In addition, an established centrifugation scheme was used, and sensitive statistical measures were applied. Thus, our results are meaningful and are applicable to routine clinical practice. Our study nevertheless has several limitations. First, lupus anticoagulant and other tests used in clinical practice were not studied, and our results can therefore not be extrapolated to these tests. Second, even though our routine centrifugation scheme $(1,500 \times g$ for 10 minutes $)$, which was used as comparator, was tested according to the CLSI guidelines, most institutions are known to use a 15-minute scheme instead. We cannot fully exclude that this might have affected our results. Third, only one centrifuge was tested, and we have not excluded that different results might have been obtained using alternative centrifuges. Thus, future investigations will seek to confirm our results in various settings using different centrifuges as well. Forth, citrated samples were 


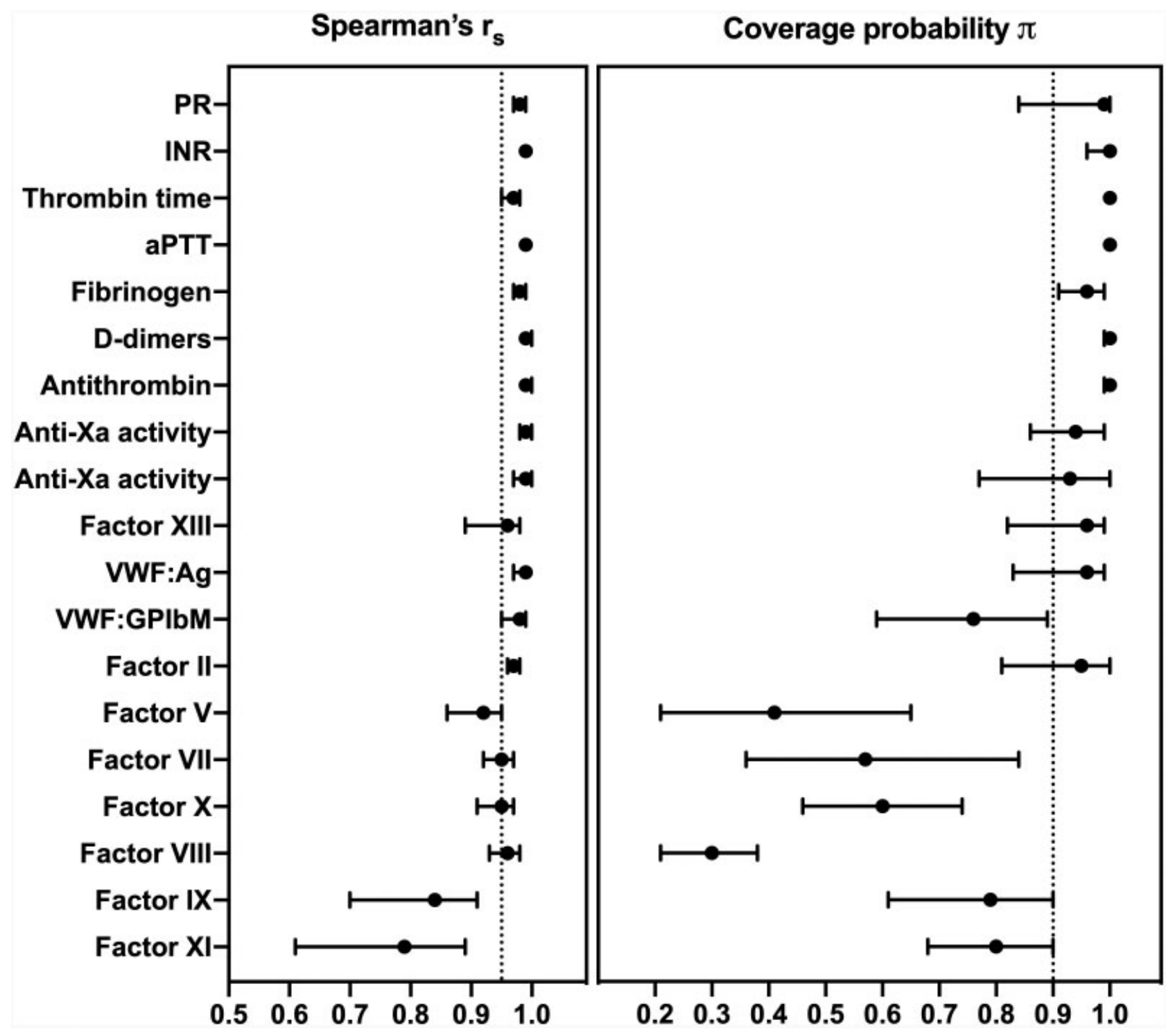

Fig. 3 Accuracy of routine hemostasis measurements obtained with a rapid, high-speed centrifugation scheme. Spearman's rank correlation coefficient $\left(r_{s}\right)$ is contrasted to the coverage probability $(C P \pi)$ at a given acceptable total deviation index $\mathrm{k}$ of 0.15 . The acceptable $r_{\mathrm{s}}$ was set at 0.95 , and the acceptable $C P \pi$ at 0.9. aPTT, activated partial thromboplastin time; INR, international normalized ratio; LMWH, low molecular weight heparin; PR, prothrombin ratio; UFH, unfractionated heparin; VWF:Ag, von Willebrand factor antigen; VWF:GPIbM, von Willebrand factor activity.

collected after EDTA samples and we cannot fully exclude that residual EDTA might have influenced the results. We believe that the risk of a bias is low because the tubes were applied to the centrifugation schemes randomly and previous studies did not demonstrate a relevant difference of standard coagulation test results. ${ }^{25}$ Fifth, for reasons of practicability not all pairs of samples were analyzed within the 2-week interval recommended by current guidelines but were transferred to a $-80^{\circ} \mathrm{C}$ freezer after several days and analyzed within 2 months. However, all pairs were handled identically, and we do not think that this might have introduced any bias. In addition, it might be argued that double centrifugation decreases preanalytical influences in case of freezing. However, aiming to compare two different (single) centrifugation schemes we did not apply double centrifugation for the purpose of this study. We do not believe that this might have introduced any bias. Seventh, plasma pathologically enriched with soluble fibrin might entrap VWF/FVIII by its complexes and technically diminish these levels under low temperature conditions. The risk of a systematic bias is low however, because both pairs of samples were treated identically.

We believe that our results add sufficient data on previous studies to justify rapid centrifugation for routine hemostasis tests (PT, thrombin time, aPTT, fibrinogen, D-dimer, antithrombin, and anti-Xa activity). Traditional centrifugation schemes shall be used for all other tests. The problem of sample identification can be solved by means of different adhesive labels ("routine" vs. "specialized hemostasis") or by means of laboratory automation procedures as done in our institution (samples with certain tests requested will be put out of the laboratory track for "manual centrifugation"). 


\section{Conclusion}

We tested the accuracy of measurements obtained with a rapid, high-speed centrifugation scheme studying a large spectrum of hemostasis tests and covering the full spectrum of values obtained in clinical practice. The new agreement measure $\mathrm{CP}$ $\pi$ identified more assays with nonconcordant results than Spearman's correlation coefficient. A clinically appropriate concordance between measurements was observed for all routine parameters, but relevant discrepancies were observed with regard to most single-factor determinations as well as von Willebrand activity. We believe that our results add sufficient data on previous studies to justify rapid centrifugation for routine hemostasis tests (PT, thrombin time, aPTT, fibrinogen, D-dimer, antithrombin, and anti-Xa activity). A traditional centrifugation scheme shall be used for all other tests.

\section{What is known about this topic?}

- Short and uniform centrifugation schemes contribute significantly to successful laboratory automation.

- To date, it is unclear if this is applicable to the hemostasis laboratory.

\section{What does this paper add?}

- We assessed a rapid, high-speed centrifugation scheme in a large set of hemostasis tests, covering the full spectrum of values obtained in clinical practice, and using meaningful statistical measures.

- We demonstrated that high-speed centrifugation is applicable to the majority of routine hemostasis parameters.

- The coverage probability was more sensitive than Spearman's rank correlation to detect disagreement among centrifugation schemes.

\section{Authors' Contributions}

N.W. contributed to study design, reviewed the analysis, and wrote the manuscript. G.G. contributed to study design and collected the data. E.G., M.R., and L.M.N. collected data. M.F. contributed to study design and provided the devices and reagents. A.B.L. contributed to study design and analysis plan and conducted the analysis. M.N. designed the study, collected data, conducted the analysis, and wrote the manuscript. All authors intellectually reviewed the manuscript.

\section{Conflict of Interest}

M.N. reports research support from Roche diagnostics, Stago, Technoclone, Pentapharm, and Siemens healthcare. G.G. reports advisory board participation for Gilead, Celgene, Takeda, Novartis, Roche, Amgen, Health Research and Consulting, Orpha Suisse. All other authors state that they have no conflict of interest.
Acknowledgments

We thank the Center of Laboratory Medicine at Inselspital for provision of reagents and devices. As the study aimed to develop routine laboratory practice in our institution, no specific external funding was obtained to conduct this work.

\section{References}

1 Genzen JR, Burnham CD, Felder RA, Hawker CD, Lippi G, Peck Palmer OM. Challenges and opportunities in implementing total laboratory automation. Clin Chem 2018;64(02):259-264

2 Hawker CD, Garr SB, Hamilton LT, Penrose JR, Ashwood ER, Weiss RL. Automated transport and sorting system in a large reference laboratory: part 1. Evaluation of needs and alternatives and development of a plan. Clin Chem 2002;48(10):1751-1760

3 Seaberg RS, Stallone RO, Statland BE. The role of total laboratory automation in a consolidated laboratory network. Clin Chem 2000;46(05):751-756

4 Lippi G, Da Rin G. Advantages and limitations of total laboratory automation: a personal overview. Clin Chem Lab Med 2019;57 (06):802-811

5 Zaninotto M, Plebani M. The "hospital central laboratory": automation, integration and clinical usefulness. Clin Chem Lab Med 2010;48(07):911-917

6 Evangelopoulos AA, Dalamaga M, Panoutsopoulos K, Dima K. Nomenclature and basic concepts in automation in the clinical laboratory setting: a practical glossary. Clin Lab 2013;59(1112):1197-1214

7 Hawker CD, Roberts WL, Garr SB, et al. Automated transport and sorting system in a large reference laboratory: part 2. Implementation of the system and performance measures over three years. Clin Chem 2002;48(10):1761-1767

8 Archetti C, Montanelli A, Finazzi D, Caimi L, Garrafa E. Clinical laboratory automation: a case study. J Public Health Res 2017;6 (01):881

9 Yu HE, Lanzoni H, Steffen T, et al. Improving laboratory processes with total laboratory automation. Lab Med 2019;50(01):96-102

10 Angeletti S, De Cesaris M, Hart JG, et al. Laboratory automation and intra-laboratory turnaround time: experience at the University Hospital Campus Bio-Medico of Rome. J Lab Autom 2015;20 (06):652-658

11 Dempfle CE, Töpfer G. Hemostaseology. In: Pre-Examination Procedures in Laboratory Diagnostics: Preanalytical Aspects and Their Impact on the Quality of Medical Laboratory Results. Berlin, Germany: De Gruyter; 2015:273-281

12 Funk D. Sample integrity and preanalytical variables. In: Quality in Laboratory Hemostasis and Thrombosis. West Sussex, United Kingdom: Wiley-Blackwell; 2013:45-56

13 Lippi G, Rossi R, Ippolito L, et al. Influence of residual platelet count on routine coagulation, factor VIII, and factor IX testing in postfreeze-thaw samples. Semin Thromb Hemost 2013;39(07): 834-839

14 Aursnes I, Vikholm V. On a possible interaction between ADP and mechanical stimulation in platelet activation. Thromb Haemost 1984;51(01):54-56

15 Magnette A, Chatelain M, Chatelain B, Ten Cate H, Mullier F. Preanalytical issues in the haemostasis laboratory: guidance for the clinical laboratories. Thromb J 2016;14:49

16 Brulé J, Revy S, Faure C, et al. Evaluation of a rapid centrifugation step ( $4500 \mathrm{~g}$ for $2 \mathrm{~min}$ ) in coagulation assays to monitor direct oral anticoagulants. Clin Chem Lab Med 2018;57(02):e37-e40

17 Boissier E, Sévin-Allouet M, Le Thuaut A, et al. A 2-min at $4500 \mathrm{~g}$ rather than a 15 -min at $2200 \mathrm{~g}$ centrifugation does not impact the reliability of 10 critical coagulation assays. Clin Chem Lab Med 2017;55(06):e118-e121

18 CLSI. Collection, Transport, and Processing of Blood Specimens for Testing Plasma-Based Coagulation Assays and Molecular Hemostasis 
Assays; Approved Guideline - Fifth Edition. In: CLSI document H21A5. Wayne, PA: Clinical and Laboratory Standards Institute; 2008

19 Polack B, Schved JF, Boneu B; Groupe d'Etude sur l'Hémostase et la Thrombose' (GEHT). Preanalytical recommendations of the 'Groupe d'Etude sur l'Hémostase et la Thrombose' (GEHT) for venous blood testing in hemostasis laboratories. Haemostasis 2001;31(01):61-68

20 Mackie I, Cooper P, Lawrie A, Kitchen S, Gray E, Laffan M; British Committee for Standards in Haematology. Guidelines on the laboratory aspects of assays used in haemostasis and thrombosis. Int J Lab Hematol 2013;35(01):1-13

21 Carroll WE, Wollitzer AO, Harris L, Ling MC, Whitaker WL, Jackson RD. The significance of platelet counts in coagulation studies. J Med 2001;32(1-2):83-96
22 Barnhart HX, Yow E, Crowley AL, et al. Choice of agreement indices for assessing and improving measurement reproducibility in a core laboratory setting. Stat Methods Med Res 2016;25(06): 2939-2958

23 Sédille-Mostafaie N, Engler H, Lutz S, Korte W. Advancing haemostasis automation-successful implementation of robotic centrifugation and sample processing in a tertiary service hospital. Clin Chem Lab Med 2013;51(06):1273-1278

24 Suchsland J, Friedrich N, Grotevendt A, et al. Optimizing centrifugation of coagulation samples in laboratory automation. Clin Chem Lab Med 2014;52(08):1187-1191

25 Indevuyst C, Schuermans W, Bailleul E, Meeus P. The order of draw: much ado about nothing? Int J Lab Hematol 2015;37(01): 50-55 